Research Article

\title{
Neuroinflammation and ALS: Transcriptomic Insights into Molecular Disease Mechanisms and Therapeutic Targets
}

\author{
Giovanna Morello, Antonio Gianmaria Spampinato, and Sebastiano Cavallaro
}

Institute of Neurological Sciences, Italian National Research Council, Catania, Italy

Correspondence should be addressed to Sebastiano Cavallaro; sebastiano.cavallaro@cnr.it

Received 20 March 2017; Revised 23 June 2017; Accepted 11 July 2017; Published 7 September 2017

Academic Editor: Thomas Möller

Copyright ( 2017 Giovanna Morello et al. This is an open access article distributed under the Creative Commons Attribution License, which permits unrestricted use, distribution, and reproduction in any medium, provided the original work is properly cited.

\begin{abstract}
Amyotrophic lateral sclerosis (ALS) is a neurodegenerative disease affecting the motor nervous system. Despite the mechanism underlying motor neuron death is not yet clarified, multiple pathogenic processes have been proposed to account for ALS. Among these, inflammatory/immune responses have recently gained particular interest, although there are conflicting reports on the role of these processes in ALS pathogenesis and treatment. This apparent discrepancy may be due to the absence of an effective stratification of ALS patients into subgroups with markedly different clinical, biological, and molecular features. Our research group recently described genome-wide characterization of motor cortex samples from sporadic ALS (SALS) patients, revealing the existence of molecular and functional heterogeneity in SALS. Here, we reexamine data coming from our previous work, focusing on transcriptomic changes of inflammatory-related genes, in order to investigate their potential contribution in ALS. A total of 1573 inflammatory genes were identified as differentially expressed between SALS patients and controls, characterizing distinct topological pathways and networks, suggestive of specific inflammatory molecular signatures for different patient subgroups. Besides providing promising insights into the intricate relationship between inflammation and ALS, this paper represents a starting point for the rationale design and development of novel and more effective diagnostic and therapeutic applications.
\end{abstract}

\section{Introduction}

Amyotrophic lateral sclerosis (ALS), or Lou Gehrig's disease, is a fatal neurodegenerative disease characterized by progressive and relatively selective degeneration of the upper and lower motor neurons in the brainstem, spinal cord, and motor cortex, resulting in paralysis and death usually within 3-5 years of diagnosis [1]. With an estimated incidence of about $1-3$ cases per 100,000 person-years and a projected lifetime risk of $1 / 2000$, ALS is considered one of the most common motor neuron diseases [2]. ALS is essentially a sporadic disorder (SALS), with $90-95 \%$ of the cases originating from an unknown cause, likely resulting from a complex gene-gene and gene-environment interplay [3-6]. The remaining $5-10 \%$ of the cases are considered familial (FALS) generally following an autosomal dominant inheritance pattern [7] due to mutations in a number of seemingly disparate genes, including SOD1 [8], ALS2 [9], SETX [10, 11], SPG11 [12], FUS [13, 14], VAPB [15], ANG [16, 17], TARDBP [18-20], FIG4 [21], OPTN [22], ATXN2 [23], and C9ORF72 [24, 25]. Interestingly, mutations in many of these genes were found in both forms, suggesting common disease mechanisms and possibly common triggers [26].

Although the pathophysiological processes and precise genetic causes underlying motor neuron death are still elusive, genomic profiling and expression studies have provided invaluable insights into the molecular mechanisms involved in ALS, supporting a "multiple-hit" hypothesis of neurodegeneration [27-35]. In this regard, a number of converging disease mechanisms are known, including oxidative damage, defective protein misfolding, mitochondrial degeneration, impaired axonal transport, neurotrophic factor deficits, apoptosis, aberrant RNA/DNA regulation, and neuroinflammation $[36,37]$. 
While ALS is not primarily considered an autoimmune or immunodeficiency disease, mounting evidence suggests that immune/inflammatory abnormalities and nonneuronal cells play an important role in the disease onset and progression. Chronically activated microglia and astrocytes as well as infiltrating immune cells represent prominent pathological findings in affected CNS areas of patients and animal models of ALS [38]. In addition, numerous anti- and proinflammatory cytokines and growth factors, including VEGF, IFN- $\gamma$, TNF- $\alpha$, IL- $1 \beta$, IL- 6 , and IL-10, seem to play a role in the neuropathological changes that characterize ALS. Nonetheless, it remains to be clarified whether neuroinflammation is a consequence of motor neuron injury or actively contributes to the development and progression of the disease. Indeed, several studies have highlighted the existence of the dual nature of inflammation in ALS, both neuroprotective and neurotoxic, that appears to be mainly dependent on the stage of disease progression. In particular, during periods of slow disease progression, the immune system exerts a protective action by secreting anti-inflammatory factors that rescue and repair damaged tissue. As the disease and motor neuron injury accelerate, a shift occurs from the beneficial immune response to a strong proinflammatory and neurotoxic state [39]. As a consequence, inflammatory mediators have received great attention as potential targets for neuroprotection in ALS, and multiple anti-inflammatory drugs (i.e., minocycline, thalidomide, celecoxib, and pioglitazone) have shown promising results in delaying disease severity in ALS animal models. Unfortunately, none of these compounds have been proved to be effective in clinical testing for ALS [40]. Reasons responsible for this failure include not only the lack of knowledge on the multiple inflammation-related events underlying ALS but also improper planning of clinical trial that does not take clinical, biological, and molecular heterogeneity of the disease into account [41]. Therefore, developing new targeted and stratified treatments that, alone or in combinations, may cope with the multiple inflammatory pathways, continue to be a research priority.

In our previous work, for the first time, we characterized unrecognized molecular heterogeneity in SALS, revealing new clues to the molecular pathogenesis and enabling the identification of novel potential predictive biomarkers and therapeutic targets that were not put in evidence by considering SALS pathology as a single entity $[42,43]$. In particular, using a computational analysis of whole genome expression profiles of 41 motor cortex samples of control (10) and SALS patients (31), we were able to differentiate SALS pathology from controls and clearly distinguish the two SALS subtypes (SALS1 and SALS2), each associated with differentially expressed genes and pathways.

In the present paper, we have reexamined our gene expression data and focused on genes and pathways that are related to inflammation. The overall goal was to assess the potential involvement of inflammatory/immunological processes in two molecularly distinct SALS patient subgroups, providing a rationale for the specific use of potential cluster-specific biomarkers and therapeutic targets.

\section{Materials and Methods}

2.1. Data Acquisition and Preprocessing. The transcriptomic data were downloaded from the ArrayExpress repository (http://www.ebi.ac.uk/arrayexpress/), with accession number E-MTAB-2325 [42]. This dataset includes transcriptional profiles of 41 postmortem motor cortex samples (31 of which were from patients with SALS and 10 were from control individuals) hybridized on the Whole Human Genome Oligo Expression Microarrays 4x44K (Agilent Technologies). The detailed information regarding clinical, genetic, and phenotypic data of patient samples has been previously described [42]. The original gene expression dataset was normalized to the 50th percentile, followed by the median baseline of all samples using GeneSpring GX v13.1 (Agilent Technologies, Italy). The resulting expression values were thresholded to $1, \log 2$ transformed, and fold changes (FCs) were calculated between the SALS patients and individual controls. Probes not corresponding to an Entrez ID were removed. In cases where several probes corresponded to one Entrez ID, the probe showing the highest variance over all samples was chosen for further analysis.

\subsection{Gene List Filtering and Differential Expression Analysis.}

The expression data were filtered to include only probes targeting genes involved in neuroinflammation and immune response. In particular, a set of inflammatory genes was generated using inflammatory/immunology-related query keywords in the Gene Ontology (GO) database (http:// www.geneontology.org/) [44]. Overall, 2637 genes were selected from the Immune system process (GO: 0002376) biological process term. This initial gene list was subjected to fold-change-based filtering and statistical analysis by using GeneSpring GX v13.1 software package (Agilent Technologies). In particular, we performed a one-way analysis of variance (ANOVA) followed by Tukey's post hoc test to identify differentially expressed genes (DEGs) between the two predefined groups of SALS patients relative to controls. Furthermore, the Benjamini-Hochberg false discovery rate (FDR) correction procedure was used to minimize falsepositive cases. An absolute FC value greater than 1.5 and an adjusted $P$ value of $<0.05$ were used as criteria for defining a set of deregulated candidate genes for further exploration.

\subsection{Construction and Topological Analysis of Protein-Protein} Interaction Network. To better clarify the interaction between immune/inflammatory DEGs and emphasize their potential contribution to ALS pathology, two extended proteinprotein interaction (PPI) networks were built by using STRING database v.10.0 and visualized with the Cytoscape v.3.4.0 software [45]. In particular, these extended networks were constructed by using DEGs in both SALS patient subgroups as seed molecules and setting a high level of confidence between molecular interactions (high confidence score of at least 0.8 ) and a maximum number of interactions to 100 . In both networks, nodes correspond to proteins encoded by DEGs in SALS, whereas edges represent the number of interactions between proteins. All interactions in both networks were unweighted and undirected. Once 


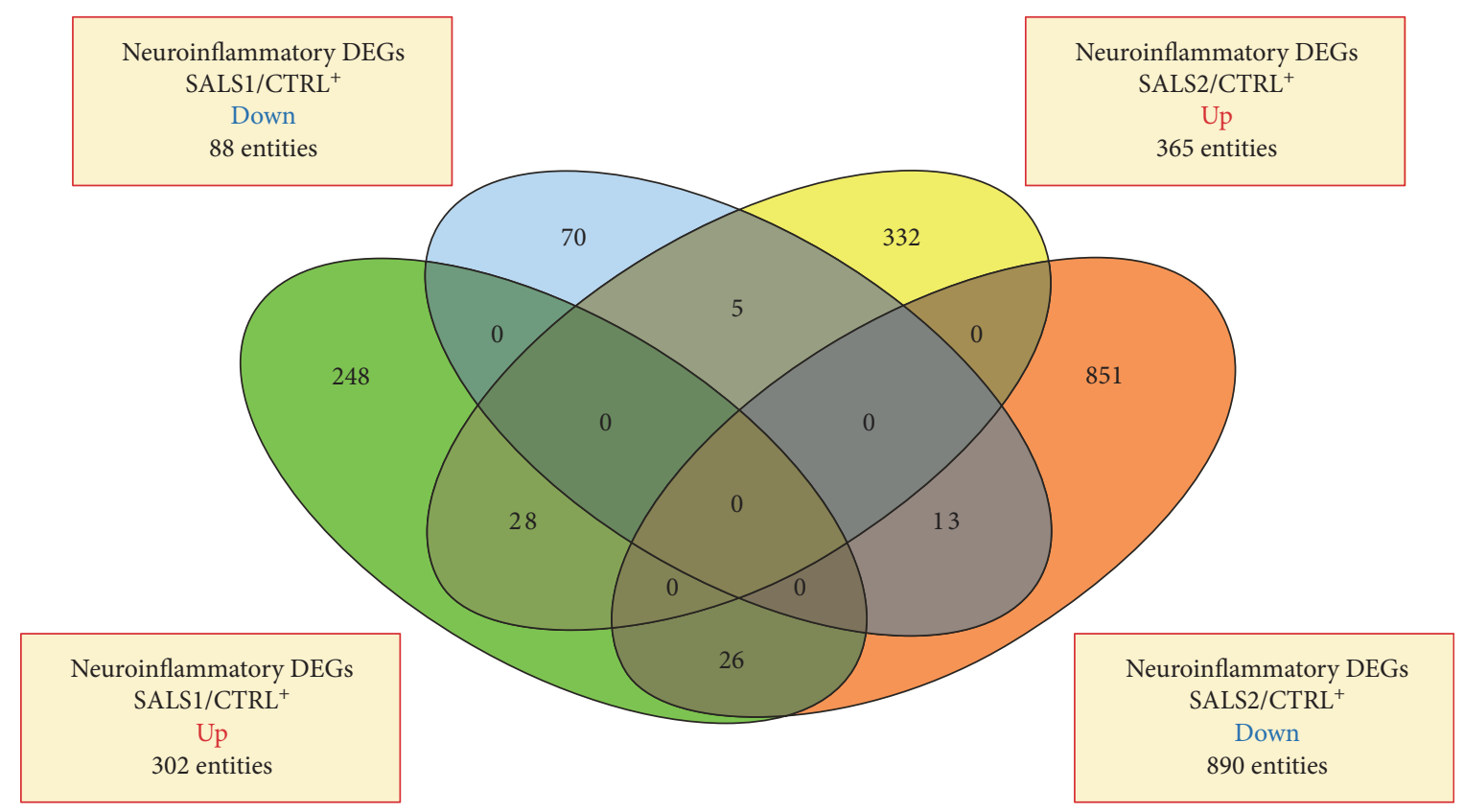

Figure 1: Venn diagrams of the total number of upregulated and downregulated inflammatory genes in the genes of SALS1 and SALS2 versus controls. Detailed information for the lists of genes differentially expressed in SALS1 and SALS2 is provided in Supplementary Tables 3-5.

extended networks were constructed, duplicated edges and self-loops were removed. Subsequently, in a prefilter process, we considered only nodes (genes) that were annotated with a high confidence score to the central nervous system by using the TISSUES web resource [46].

In order to identify the "hub" nodes, a network topology analysis was performed by using the Cytoscape plug-in NetworkAnalyzer based on topological parameters [47]. Node (gene) centrality in both networks was also investigated through the evaluation of "node degree." This topological parameter indicates the relevance of a gene (node) as functionally capable of holding together the communicating nodes in a biological network. Nodes with high degree (hub genes) represented the genes having important biological functions: the higher the value, the higher the relevance of the gene in connecting regulatory molecules. The final PPI networks were visualized based on node degree and edge betweenness parameters. The relative importance of the genes in each network was determined based on the node centrality measure setting the topological parameter "node degree" $\geq 10$. Likewise, values of edge betweenness were mapped with the edge size: high values of this parameter correspond to a large edge size.

2.4. Functional Analysis and Selection of the Candidate Pathways. To investigate the relevance of inflammatoryrelated DEGs in determining specific molecular signatures in SALS pathology, pathway analysis was performed with Ingenuity Pathway Analysis (IPA ${ }^{\circledR}$; http://www.ingenuity. $\mathrm{com} /$ ) and GeneGO MetaCore ${ }^{\mathrm{TM}}$ [48]. Both these programs identify significantly enriched biological pathways and signaling cascades that are associated with a given list of genes by calculating the hypergeometric distribution. In accordance with the purpose of this study, we focused on predefined the "canonical pathways" commonly associated with immune response/inflammation processes. Finally, immune/inflammatory pathways with a $P$ value of $<0.05$ and a fold change of $>1.5$ were screened and analyzed.

In addition, to reduce potential errors due to the use of preselected gene sets [49] and to increase the strength of the functional analysis, we also performed a "control" pathway enrichment analysis both on the entire list of differentially expressed genes in SALS patients versus controls, both without the assignment of SALS patients into the two cluster groups (see Supplementary Tables 1 and 2 available online at https://doi.org/10.1155/2017/7070469).

\section{Results}

3.1. Identification of Differentially Expressed Inflammatory Genes. Our results indicate that 1573 out of 2637 inflammation genes (probes) were differentially expressed between SALS patients and controls. In particular, a total of 390 immune/inflammation genes were found to be significantly differentially expressed in SALS1 (302 upregulated and 88 downregulated), while SALS2 patients showed significant changes of expression in 1255 genes (365 upregulated and 890 downregulated) (Figure 1; Supplementary Tables 3-5). Although some of these genes (72) were differentially expressed in both pairwise comparisons, the majority of them were cluster-specific (Figure 1).

3.2. Network Analysis and Characterization of Hub Genes. To gain further insights into the functional significance of inflammatory-related DEGs in SALS and prioritize putative genetic markers that might increase the susceptibility of patients affected by SALS, we mapped these genes into PPI networks for each SALS subgroup. Initially, the extended 


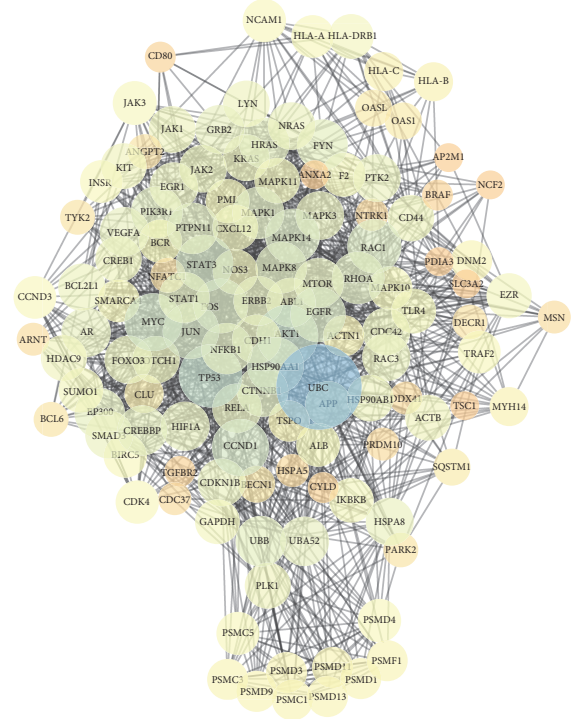

(a)

\begin{tabular}{|c|c|c|c|}
\hline$\frac{D \text { Degree }}{\text { Def }}$ & Node & Degree & Node \\
\hline & UBC & 40 & ERBB2 \\
\hline 84 & TP533 & 40 & MTOR \\
\hline 79 & AKT1 & 39 & ABLI \\
\hline 66 & MYC & 39 & $\mathrm{AR}$ \\
\hline 64 & CCND1 & 39 & RELA \\
\hline 64 & STAT3 & 39 & VEGFA \\
\hline 63 & HSP9OAA1 & 39 & UBA52 \\
\hline 62 & JUN & 38 & CDH1 \\
\hline 58 & EGFR & 38 & GRB2 \\
\hline 56 & FOS & 38 & HIFIA \\
\hline 56 & MAPK14 & 36 & EGR1 \\
\hline 54 & RAC1 & 36 & МАРКЗ \\
\hline 49 & RHOA & 35 & $\mathrm{RAC3}$ \\
\hline 47 & MAPK1 & 34 & Foxo3 \\
\hline 47 & PIK3R1 & 34 & HSPAB \\
\hline 46 & MAPK8 & 34 & CDKN1B \\
\hline 45 & NFKB1 & 34 & SMAD3 \\
\hline 45 & CTNNB1 & 33 & JAK2 \\
\hline 45 & PTPN11 & 33 & NRAS \\
\hline 44 & FYN & 33 & $\mathrm{CDC42}$ \\
\hline 44 & STAT1 & 33 & PTK2 \\
\hline 43 & HRAS & 32 & оттнн \\
\hline 43 & UBB & 32 & BCL2L1 \\
\hline 42 & EP300 & 30 & \\
\hline 41 & CREBBP & 30 & IYN \\
\hline
\end{tabular}

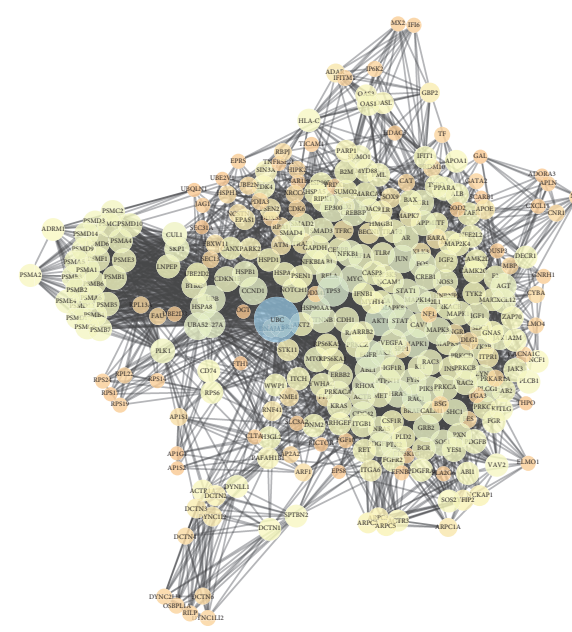

\begin{tabular}{lccc}
\hline Degree & Node & Degree & Node \\
\hline 326 & UBC & 70 & STAT1 \\
131 & AKT1 & 69 & ABL1 \\
130 & TP53 & 69 & FYN \\
105 & EGFR & 68 & RELA \\
99 & RAC1 & 68 & JAK2 \\
98 & STAT3 & 68 & CREBBP \\
97 & MAPK1 & 67 & CDC42 \\
95 & HSP90AA1 & 67 & VEGFA \\
90 & JUN & 66 & EP300 \\
89 & MYC & 66 & SOS1 \\
88 & CCND1 & 65 & HSPA8 \\
87 & UBB & 64 & SHC1 \\
86 & RPS27A & 62 & AGT \\
81 & HRAS & 61 & PTK2 \\
81 & UBA52 & 61 & HSPB1 \\
79 & MAPK14 & 60 & CREB1 \\
78 & RHOA & 59 & PLCG1 \\
76 & CTNNB1 & 57 & AR \\
76 & MAPK8 & 56 & RAC3 \\
75 & PIK3R1 & 56 & CDH1 \\
75 & MAPK3 & 56 & EGR1 \\
74 & PTPN11 & 56 & CDKN1B \\
74 & FOS & 55 & NRAS \\
72 & NFKB1 & 55 & PRKCA \\
71 & GRB2 & 55 & RAF1 \\
\hline
\end{tabular}

(b)

FIgure 2: SALS-related PPI networks. (a) Graphical representation of the PPI network from inflammatory DEGs in SALS1. Nodes having a high degree are the ones that form most interactions with other nodes and were displayed as a big circle and dark colors. The right panel shows the top 50 nodes in PPI network order by descending degree value. (b) Graphical representation of the PPI network from inflammatory DEGs in SALS2. Nodes having a high degree are the ones that form most interactions with other nodes and were displayed as a big circle and dark colors. The right panel shows the top 50 nodes in PPI network order by descending degree value.

PPI network for SALS1 contained 448 gene signatures (nodes) with 25,512 interactions (edges), while the extended PPI network associated with SALS2 had 1123 nodes and 8667 interactions. Identifying hub genes as the DEGs with the highest node degree and ranking them for importance based on the node centrality measure reduced the SALS1-PPI network to 126 nodes connected through 1160 edges (with high values of betweenness) and the SALS2-PPI network to 327 nodes connected through 4867 edges (Figure 2 and Supplementary Tables 6 and 7). The information corresponding to the centralities of the top 50 ranked genes in both networks is represented in Figure 2.

Integrated analysis revealed that the two SALS-PPI networks shared similar genetic nodes and interactions. Although both, driving regulators and essential genes, are profoundly different between SALS patient subgroups, they are complementary and converge to similar immune/inflammatory signaling mechanisms within their respective subtypes (Figure 2). In particular, it was observed that $U B C$ was the top-ranked gene within PPI networks for both SALS1 and SALS2 patients, with node degrees of 157 and 326, respectively, and thus constituted a superhub gene with a wide variety of cooperative partners. These data indicated that $U B C$, together with other key nodes (hubs) displaying the highest connectivity in both networks, such as AKT1 and TP53, may play a critical role in activating the neuroinflammatory state in SALS patients and thus may represent potential genetic markers with direct or indirect involvement in ALS pathogenesis.

3.3. Functional Enrichment Analysis of Inflammatory DEGs in SALS Patient Subgroups. In order to better characterize specific inflammatory molecular signatures for
SALS, we investigated whether DE inflammatory genes in SALS patients were enriched for certain specific biological functions and pathways, by using functional ontologies represented in IPA and MetaCore repositories (see Materials and Methods).

Comparison of both SALS patient subtypes with the total control group revealed a total of 585 significantly deregulated pathways, the majority of which were deregulated in the opposite way in the two SALS subtypes (Figure 3 and Supplementary Table 8). In particular, SALS1 was mainly characterized by increased expression of genes involved in the inflammatory response, including complement system and antigen presentation pathway $(P$ value $=1.082 E-14)$, chemokines and cell adhesion $(P$ value $=2.649 E-07)$, and cytoskeletal remodeling $(P$ value $=9.824 E-07)$, as well as a reduced expression of genes associated with the apoptotic signaling $(P$ value $=1.145 E-02)$. SALS2 patients, instead, showed an overall downregulation of inflammatoryrelated pathways, such as $H M G B 1 / R A G E$ signaling pathway $(P$ value $=1.203 E-13)$ and $B$ cell antigen receptor pathway $(P$ value $=5.132 E-13)$ as well as alteration of oxidative stress $(P$ value $=2.133 E-13)$. A detailed description of the most significant variations implicated in the inflammatory and immunological pathways affected in SALS is provided in the Supplementary Information section (Supplementary Discussion and Supplementary Figures 1-4).

\section{Discussion}

Inflammation and abnormal or hyperactive immune responses play a pivotal role in the pathogenesis and progression of several neurodegenerative diseases, including ALS. While the molecular basis of neuroinflammation in 


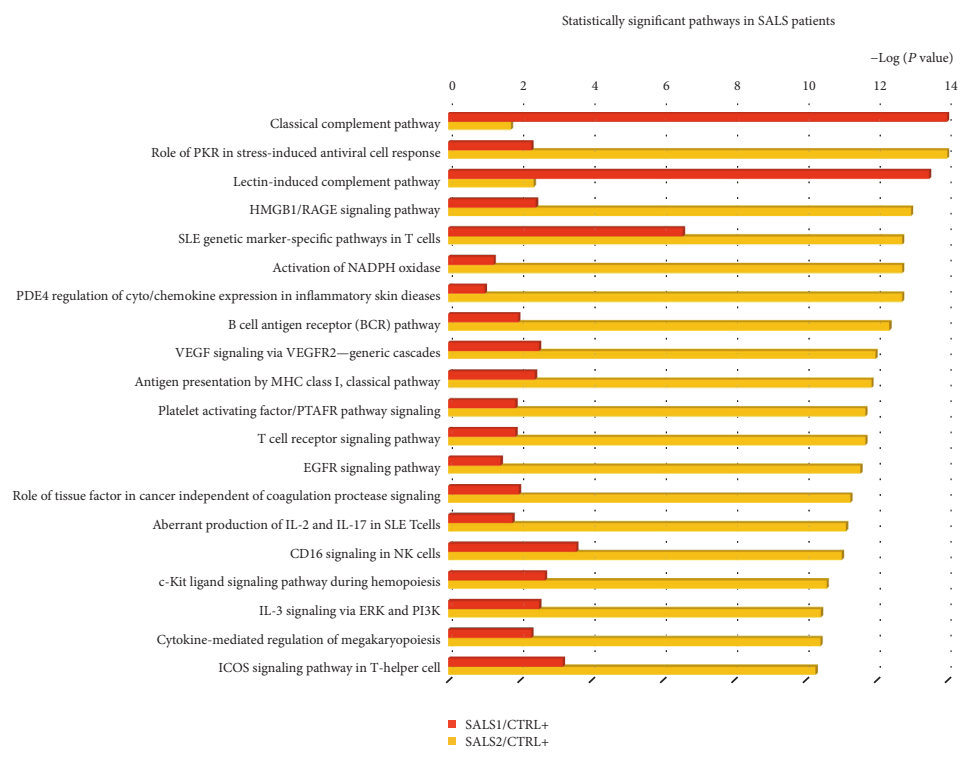

(a)

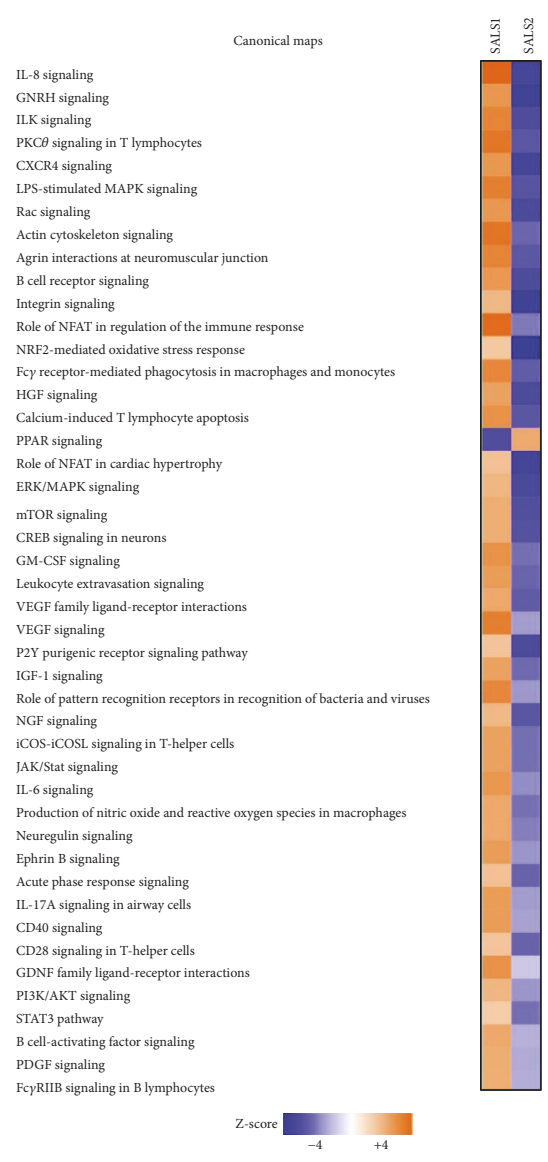

(b)

FIgURE 3: Functional enrichment analysis of inflammatory DEGs in SALS patients. (a) Representation of the top 20 most significantly enriched $(P$ value $<0.05)$ canonical pathway maps associated with the neuroinflammatory DEGs genes in both SALS patient subgroups when compared to controls. A histogram of statistical significance ( $-\log P$ value) is shown: the list is arranged in descending order with the most significant pathways at the top. The analysis was performed using the MetaCore pathway analysis suite. (b) The heat map from IPA of canonical signaling pathways (rows) most significantly enriched in neuroinflammatory genes is differentially expressed in the two SALS patient subgroups (columns). The score magnitudes are shown in a gradient color, from light to bright orange, for induced, and from light to bright blue, for suppressed pathway activity. Detailed information about pathway map enrichment analysis is described in Supplementary Table 8 .

ALS are being defined, the tools and indicators for early diagnosis and effective treatment options remain incompletely characterized as well as their complex interplay within the major signaling cascades occurring during neuroinflammatory processes. Multiple anti-inflammatory compounds have been evaluated preclinically for their therapeutic potential in ALS showing promising results, but none of these have been proved to be effective in patients [40]. This failure may be mainly due to the absence of an effective stratification of ALS patients into subgroups with markedly different clinical, biological, and molecular features [41].

In the current study, we reanalyzed our previous dataset (E-MTAB-2325), consisting of whole genome expression profiles of 41 motor cortex samples from SALS and control patients, focusing on transcriptomic changes of multiple genes involved in various aspects of inflammation and immune responses, in order to investigate their potential contribution in SALS etiopathogenesis.
Although the use of postmortem brain tissues impedes deeper understanding of the pathophysiological processes ongoing in the diseased brain, they represent a valuable resource for human studies, providing valuable information that cannot be obtained by using other approaches on a living patient.

A total of 1573 inflammatory genes were differentially expressed between SALS patients and controls, the majority of which were cluster-specific, suggestive of a great divergence of the two SALS subgroups at the molecular level (Figure 1).

Components of inflammatory/immune responses are very numerous and interact with each other across multiple functional pathways, impeding the identification of genetic risk factors that effectively contribute to the neuroinflammatory process in ALS. To prioritize the identification of key molecular candidates that could be used for the discovery of therapeutic targets and diagnostic biomarkers, we performed a network analysis on shortlists of inflammatory 
DEGs in SALS patients (Figure 2). This machine learningbased approach revealed that, although profoundly different, the two subtype-specific SALS networks were complementary and converged to similar immune/inflammatory signaling mechanisms and driving genes, suggesting that in each SALS subtype, there is a "deterministic" path for aberrant immune/ inflammatory responses driven by genomic alterations, and the networks could, therefore, provide "predictable" power for selective genomic alterations. In particular, UBC was identified as a superhub gene within both SALS-related PPI networks (Figures 2(a) and 2(b)). These findings are in line with the results of a previous network-based genomic analysis on peripheral motor nerves of SALS patients, which revealed a significant overrepresentation of pathways related to ubiquitin-protein ligase activity and identified $U B C$ as the most relevant hub gene [50]. The functional significance and contribution of ubiquitin in ALS pathology are also supported by the presence of low expression levels and cytoplasmic inclusions of this protein in the spinal cord motor neurons of ALS patients, supporting its potential role as a biomarker for the disease [51-54]. Despite pharmacologic strategies, aimed to increase or replace the specific lost ubiquitin activity, that have been demonstrated to be effective in preventing abnormal protein accumulation in several human disorders, the clinical use of these treatments has proved to be particularly challenging due to their numerous potential off-target effects [55]. Therefore, there is a need to design therapies that selectively interfere with various components of the ubiquitin-proteasome system, offering new therapeutic perspectives for the treatment of various neurological diseases, including ALS.

To investigate the combined effects of multiple immune/ inflammation dysregulations, we have searched for canonical signaling pathways significantly enriched in inflammatory DEGs and mapped them into IPA and MetaCore repositories of signaling pathways (Figures 3(a) and 3(b)). Functional classification of these DEGs showed that antigen processing and presentation, complement system, and reactive oxygen species production are the most significant immune/ inflammation pathways deregulated in SALS, suggesting that these processes play a crucial role in the progressive degeneration and loss of motor neurons (Figure 3(a)). Scientific evidence about a pathogenic role for most of these pathways is already available in the literature [41, 56-60]; however, the contribution of the single inflammatory cascade to the distinct SALS subtypes still needs to be clarified. Notably, the majority of the identified signaling cascades were deregulated in the opposite way in the two SALS subtypes, supporting the existence of a specific molecular signature associated with the immune/inflammatory status in SALS patients (Figure 3(b)). In particular, SALS1 patients seem to be associated with an increased inflammatory phenotype while SALS2 patients show reduced expression levels in genes involved in immune response and inflammatory signaling pathways.

Interestingly, the analysis of deregulated inflammatory cascades reveals the involvement of a variety of genes that have been implicated, up to date, in the causation and/or susceptibility of ALS (Supplementary Tables 3 and 4). Among these, one of the striking observations is the differential expression of numerous ALS-linked genes (i.e., ANG, DCTN1, SQSTM1, and TBK1) involved in autophagy, a highly conserved and tightly regulated cellular selfdegradative process whose alteration leads to an impaired clearance of toxic protein aggregates and/or of damaged mitochondria that represent some of the best characterized hallmarks of both SALS and FALS [61]. In particular, significantly reduced mRNA levels of TBK1, the most recently identified ALS gene, were observed in SALS2, confirming that reduced activity of this enzyme may result in impaired autophagy and contribute to the accumulation of protein aggregates in motor neurons and ALS pathology [62]. Given the implication that TBK1 plays a key role in ALS and the observation that some autophagy inducers, such as rapamycin, have been already shown to be promising ALS drug candidates, it seems worthwhile to explore TBK1 as a more defined target as well as envisage the use of its pharmacological activators for developing novel and targeted therapeutics for patients.

Other than confirming the role of previously ALS-linked genes, our analysis also identified novel potential candidate genes that deserve further investigation and validation for better establishing their role in ALS pathology. Among these, we distinguish the deregulated expression of a series of molecules implicated in antigen generation and/or trimming, sustaining the involvement of a dysfunction in protein turnover and ubiquitin-proteasome pathways in ALS (Supplementary Figure 1). In particular, overexpression of IMPAS-1 in SALS patients is supported by several studies that correlate high levels of this protease with the aberrant autophagic activity associated with numerous neurodegenerative diseases [63]. All together, these results suggest that deciphering the complex actions of altered protein recycling and degradation machinery networks may help to further elucidate the neuroinflammatory processes occurring in ALS.

\section{Conclusion}

Overall, our findings not only provide interesting insights into the role of inflammatory/immune responses in the pathogenesis of SALS but also underline the existence of molecular heterogeneity in the inflammatory status of different subtypes of SALS patients, providing a rationale for the specific use of potential cluster-specific biomarkers and therapeutic targets. However, it is necessary to take into consideration that deregulation of identified candidate genes in human postmortem tissues may be due to reactive changes that occur in the final stages of disease, impeding to distinguish causative factors from secondary degenerative changes ongoing in the diseased brain. Therefore, future functional and clinical investigation will be necessary to assess the potential role of these candidates in affecting the origins and/or progression of the disease, opening the way to the development of novel and more effective diagnostic, prognostic, and therapeutic applications. 


\section{Abbreviations}

SOD1: $\quad$ Superoxide dismutase 1

ALS2: Alsin

SETX: Senataxin

SPG11: $\quad$ Spastic paraplegia 11

FUS: $\quad$ Fused in sarcoma RNA binding protein

VAMP: Vesicle-associated membrane protein

VAPB: Associated protein B and C

ANG: Angiogenin

TARDBP: TAR DNA-binding protein

FIG4: $\quad$ FIG4 phosphoinositide 5-phosphatase

OPTN: Optineurin

ATXN2: Ataxin-2

C9ORF72: Chromosome 9 open reading frame 72

VEGF: Vascular endothelial growth factor

IFN- $\gamma$ : Interferon gamma

TNF- $\alpha$ : Tumor necrosis factor

ILs: Interleukins

IL-1 $\beta$ : Interleukin 1 beta

UBC: $\quad$ Ubiquitin $C$

TBK1: $\quad$ TANK-binding kinase 1

SQSTM1: Sequestosome 1

DCTN1: Dynactin subunit 1

IMPAS-1: Histocompatibility minor 13.

\section{Conflicts of Interest}

The authors declare no competing financial interests.

\section{Authors' Contributions}

Giovanna Morello wrote the manuscript. Antonio Gianmaria Spampinato performed the bioinformatics analyses. Sebastiano Cavallaro conceived, directed, and supervised the project. All authors have read and approved the final version of this manuscript and agreed to be accountable for all aspects of the work.

\section{Acknowledgments}

This work was supported by the Italian Ministry of Education, Universities and Research through Grant no. CTN01_00177_817708. The authors gratefully acknowledge Cristina Calì, Alfia Corsino, Maria Patrizia D’Angelo, and Francesco Marino for their administrative and technical assistance.

\section{References}

[1] J. D. Mitchell and G. D. Borasio, "Amyotrophic lateral sclerosis," Lancet, vol. 369, no. 9578, pp. 2031-2041, 2007.

[2] A. Eisen, "Amyotrophic lateral sclerosis-evolutionary and other perspectives," Muscle \& Nerve, vol. 40, no. 2, pp. 297304, 2009.

[3] C. L. Simpson and A. Al Chalabi, "Amyotrophic lateral sclerosis as a complex genetic disease," Biochimica et Biophysica Acta (BBA) - Molecular Basis of Disease, vol. 1762, no. 11-12, pp. 973-985, 2006.
[4] P. M. Andersen and A. Al-Chalabi, "Clinical genetics of amyotrophic lateral sclerosis: what do we really know?," Nature Reviews Neurology, vol. 7, no. 11, pp. 603-615, 2011.

[5] C. Armon, "Environmental risk factors for amyotrophic lateral sclerosis," Neuroepidemiology, vol. 20, no. 1, pp. 2-6, 2001.

[6] D. Majoor-Krakauer, P. J. Willems, and A. Hofman, "Genetic epidemiology of amyotrophic lateral sclerosis," ClinicalGenetics, vol. 63, no. 2, pp. 83-101, 2003.

[7] P. G. Ince, J. R. Highley, J. Kirby et al., "Molecular pathology and genetic advances in amyotrophic lateral sclerosis: an emerging molecular pathway and the significance of glial pathology," Acta Neuropathologica, vol. 122, no. 6, pp. 657$671,2011$.

[8] D. R. Rosen, "Mutations in Cu/Zn superoxide dismutase gene are associated with familial amyotrophic lateral sclerosis," Nature, vol. 364, no. 6435, p. 362, 1993.

[9] S. Hadano, C. K. Hand, H. Osuga et al., "A gene encoding a putative GTPase regulator is mutated in familial amyotrophic lateral sclerosis 2," Nature Genetics, vol. 29, no. 2, pp. 166-173, 2001.

[10] Y. Z. Chen, C. L. Bennett, H. M. Huynh et al., "DNA/RNA helicase gene mutations in a form of juvenile amyotrophic lateral sclerosis (ALS4)," The American Journal of Human Genetics, vol. 74, no. 6, pp. 1128-1135, 2004.

[11] P. F. Chance, B. A. Rabin, S. G. Ryan et al., "Linkage of the gene for an autosomal dominant form of juvenile amyotrophic lateral sclerosis to chromosome 9q34," The American Journal of Human Genetics, vol. 62, no. 3, pp. 633-640, 1998.

[12] A. Orlacchio, C. Babalini, A. Borreca et al., "SPATACSIN mutations cause autosomal recessive juvenile amyotrophic lateral sclerosis," Brain, vol. 133, Part 2, pp. 591-598, 2010.

[13] C. Vance, B. Rogelj, T. Hortobagyi et al., "Mutations in FUS, an RNA processing protein, cause familial amyotrophic lateral sclerosis type 6," Science, vol. 323, no. 5918, pp. 1208-1211, 2009.

[14] T. J. Kwiatkowski Jr., D. A. Bosco, A. L. Leclerc et al., "Mutations in the FUS/TLS gene on chromosome 16 cause familial amyotrophic lateral sclerosis," Science, vol. 323, no. 5918, pp. 1205-1208, 2009.

[15] A. L. Nishimura, M. Mitne-Neto, H. C. Silva et al., "A mutation in the vesicle-trafficking protein VAPB causes late-onset spinal muscular atrophy and amyotrophic lateral sclerosis," The American Journal of Human Genetics, vol. 75, no. 5, pp. 822-831, 2004.

[16] M. J. Greenway, P. M. Andersen, C. Russ et al., “ANG mutations segregate with familial and 'sporadic' amyotrophic lateral sclerosis," Nature Genetics, vol. 38, no. 4, pp. 411-413, 2006.

[17] H. J. Chen, G. Anagnostou, A. Chai et al., "Characterization of the properties of a novel mutation in VAPB in familial amyotrophic lateral sclerosis," The Journal of Biological Chemistry, vol. 285, no. 51, pp. 40266-40281, 2010.

[18] J. Sreedharan, I. P. Blair, V. B. Tripathi et al., "TDP-43 mutations in familial and sporadic amyotrophic lateral sclerosis," Science, vol. 319, no. 5870, pp. 1668-1672, 2008.

[19] M. A. Gitcho, R. H. Baloh, S. Chakraverty et al., "TDP-43 A315T mutation in familial motor neuron disease," Annals of Neurology, vol. 63, no. 4, pp. 535-538, 2008.

[20] E. Kabashi, P. N. Valdmanis, P. Dion et al., "TARDBP mutations in individuals with sporadic and familial amyotrophic lateral sclerosis," Nature Genetics, vol. 40, no. 5, pp. 572-574, 2008. 
[21] C. Y. Chow, J. E. Landers, S. K. Bergren et al., "Deleterious variants of FIG4, a phosphoinositide phosphatase, in patients with ALS," American Journal of Human Genetics, vol. 84, no. 1, pp. 85-88, 2009.

[22] M. A. van Es, F. P. Diekstra, J. H. Veldink et al., "A case of ALSFTD in a large FALS pedigree with a K17I ANG mutation," Neurology, vol. 72, no. 3, pp. 287-288, 2009.

[23] A. C. Elden, H. J. Kim, M. P. Hart et al., "Ataxin-2 intermediate-length polyglutamine expansions are associated with increased risk for ALS," Nature, vol. 466, no. 7310, pp. 1069-1075, 2010.

[24] M. DeJesus-Hernandez, I. R. Mackenzie, B. F. Boeve et al., "Expanded GGGGCC hexanucleotide repeat in noncoding region of C9ORF72 causes chromosome 9p-linked FTD and ALS," Neuron, vol. 72, no. 2, pp. 245-256, 2011.

[25] A. E. Renton, E. Majounie, A. Waite et al., "A hexanucleotide repeat expansion in C9ORF72 is the cause of chromosome 9p21-linked ALS-FTD," Neuron, vol. 72, no. 2, pp. 257-268, 2011.

[26] S. Guareschi, E. Cova, C. Cereda et al., “An over-oxidized form of superoxide dismutase found in sporadic amyotrophic lateral sclerosis with bulbar onset shares a toxic mechanism with mutant SOD1," Proceedings of the National Academy of Sciences of the United States of America, vol. 109, no. 13, pp. 5074-5079, 2012.

[27] S. Cluskey and D. B. Ramsden, "Mechanisms of neurodegeneration in amyotrophic lateral sclerosis," Molecular Pathology, vol. 54, no. 6, pp. 386-392, 2001.

[28] A. Malaspina, N. Kaushik, and J. de Belleroche, "Differential expression of 14 genes in amyotrophic lateral sclerosis spinal cord detected using gridded cDNA arrays," Journal of Neurochemistry, vol. 77, no. 1, pp. 132-145, 2001.

[29] Y. M. Jiang, M. Yamamoto, Y. Kobayashi et al., "Gene expression profile of spinal motor neurons in sporadic amyotrophic lateral sclerosis," Annals of Neurology, vol. 57, no. 2, pp. 236-251, 2005.

[30] F. Dangond, D. Hwang, S. Camelo et al., "Molecular signature of late-stage human ALS revealed by expression profiling of postmortem spinal cord gray matter," Physiological Genomics, vol. 16, no. 2, pp. 229-239, 2004.

[31] D. Offen, Y. Barhum, E. Melamed, N. Embacher, C. Schindler, and G. Ransmayr, "Spinal cord mRNA profile in patients with ALS: comparison with transgenic mice expressing the human SOD-1 mutant," Journal of Molecular Neuroscience, vol. 38, no. 2, pp. 85-93, 2009.

[32] X. S. Wang, Z. Simmons, W. Liu, P. J. Boyer, and J. R. Connor, "Differential expression of genes in amyotrophic lateral sclerosis revealed by profiling the post mortem cortex," Amyotrophic Lateral Sclerosis, vol. 7, no. 4, pp. 201-210, 2006.

[33] C. W. Lederer, A. Torrisi, M. Pantelidou, N. Santama, and S. Cavallaro, "Pathways and genes differentially expressed in the motor cortex of patients with sporadic amyotrophic lateral sclerosis," BMC Genomics, vol. 8, no. 1, p. 26, 2007.

[34] S. J. Rabin, J. M. Kim, M. Baughn et al., "Sporadic ALS has compartment-specific aberrant exon splicing and altered cell-matrix adhesion biology," Human Molecular Genetics, vol. 19, no. 2, pp. 313-328, 2010.

[35] L. E. Cox, L. Ferraiuolo, E. F. Goodall et al., "Mutations in CHMP2B in lower motor neuron predominant amyotrophic lateral sclerosis (ALS)," PLoS One, vol. 5, no. 3, article e9872, 2010 .
[36] S. Morgan and R. W. Orrell, "Pathogenesis of amyotrophic lateral sclerosis," British Medical Bulletin, vol. 119, no. 1, pp. 87-98, 2016.

[37] W. Robberecht and T. Philips, "The changing scene of amyotrophic lateral sclerosis," Nature Reviews Neuroscience, vol. 14 , no. 4 , pp. $248-264,2013$

[38] P. A. McCombe and R. D. Henderson, "The role of immune and inflammatory mechanisms in ALS," Current Molecular Medicine, vol. 11, no. 3, pp. 246-254, 2011.

[39] K. G. Hooten, D. R. Beers, W. Zhao, and S. H. Appel, "Protective and toxic neuroinflammation in amyotrophic lateral sclerosis," Neurotherapeutics: the Journal of the American Society for Experimental NeuroTherapeutics, vol. 12, no. 2, pp. 364-375, 2015.

[40] F. Rizzo, G. Riboldi, S. Salani et al., "Cellular therapy to target neuroinflammation in amyotrophic lateral sclerosis," Cellular and Molecular Life Sciences: CMLS, vol. 71, no. 6, pp. 999-1015, 2014.

[41] A. Malaspina, F. Puentes, and S. Amor, "Disease origin and progression in amyotrophic lateral sclerosis: an immunology perspective," International Immunology, vol. 27, no. 3, pp. 117-129, 2015.

[42] E. Aronica, F. Baas, A. Iyer, A. L. ten Asbroek, G. Morello, and S. Cavallaro, "Molecular classification of amyotrophic lateral sclerosis by unsupervised clustering of gene expression in motor cortex," Neurobiology of Disease, vol. 74, pp. 359-376, 2015.

[43] G. Morello and S. Cavallaro, "Transcriptional analysis reveals distinct subtypes in amyotrophic lateral sclerosis: implications for personalized therapy," Future Medicinal Chemistry, vol. 7, no. 10, pp. 1335-1359, 2015.

[44] M. A. Harris, J. Clark, A. Ireland et al., "The Gene Ontology (GO) database and informatics resource," Nucleic Acids Research, vol. 32, Database issue, pp. D258-D261, 2004.

[45] D. Szklarczyk, A. Franceschini, S. Wyder et al., "STRING v10: protein-protein interaction networks, integrated over the tree of life," Nucleic Acids Research, vol. 43, Database issue, pp. D447-D452, 2015.

[46] A. Santos, K. Tsafou, C. Stolte, S. Pletscher-Frankild, S. I. O'Donoghue, and L. J. Jensen, "Comprehensive comparison of large-scale tissue expression datasets," PeerJ, vol. 3, article e1054, 2015.

[47] Y. Assenov, F. Ramirez, S. E. Schelhorn, T. Lengauer, and M. Albrecht, "Computing topological parameters of biological networks," Bioinformatics, vol. 24, no. 2, pp. 282-284, 2008.

[48] Y. Nikolsky, S. Ekins, T. Nikolskaya, and A. Bugrim, “A novel method for generation of signature networks as biomarkers from complex high throughput data," Toxicology Letters, vol. 158 , no. 1 , pp. $20-29,2005$.

[49] T. Bleazard, J. A. Lamb, and S. Griffiths-Jones, "Bias in microRNA functional enrichment analysis," Bioinformatics, vol. 31, no. 10, pp. 1592-1598, 2015.

[50] N. Riva, F. Clarelli, T. Domi et al., "Unraveling gene expression profiles in peripheral motor nerve from amyotrophic lateral sclerosis patients: insights into pathogenesis," Scientific Reports, vol. 6, article 39297, 2016.

[51] A. A. Bergen, S. Kaing, J. B. ten Brink, B. Netherlands Brain, T. G. Gorgels, and S. F. Janssen, "Gene expression and functional annotation of human choroid plexus epithelium failure in Alzheimer's disease," BMC Genomics, vol. 16, p. 956, 2015. 
[52] L. Petrucelli and T. M. Dawson, "Mechanism of neurodegenerative disease: role of the ubiquitin proteasome system," Annals of Medicine, vol. 36, no. 4, pp. 315-320, 2004.

[53] C. A. Ross and M. A. Poirier, "Protein aggregation and neurodegenerative disease," Nature Medicine, Supplement 10, pp. S10-S17, 2004.

[54] J. Hanrieder, T. Ekegren, M. Andersson, and J. Bergquist, "MALDI imaging of post-mortem human spinal cord in amyotrophic lateral sclerosis," Journal of Neurochemistry, vol. 124, no. 5, pp. 695-707, 2013.

[55] N. L. Lehman, "The ubiquitin proteasome system in neuropathology," Acta Neuropathologica, vol. 118, no. 3, pp. 329-347, 2009.

[56] G. P. de Oliveira, C. J. Alves, and G. Chadi, "Early gene expression changes in spinal cord from SOD1 (G93A) amyotrophic lateral sclerosis animal model," Frontiers in Cellular Neuroscience, vol. 7, p. 216, 2013.

[57] C. A. Lewis, J. Manning, F. Rossi, and C. Krieger, "The neuroinflammatory response in ALS: the roles of microglia and T cells," Neurology Research International, vol. 2012, Article ID 803701, 8 pages, 2012.

[58] N. Bahia El Idrissi, S. Bosch, V. Ramaglia, E. Aronica, F. Baas, and D. Troost, "Complement activation at the motor endplates in amyotrophic lateral sclerosis," Journal of Neuroinflammation, vol. 13, no. 1, p. 72, 2016.

[59] M. Rentzos, A. Rombos, C. Nikolaou et al., "Interleukin-17 and interleukin-23 are elevated in serum and cerebrospinal fluid of patients with ALS: a reflection of Th17 cells activation?," Acta Neurologica Scandinavica, vol. 122, no. 6, pp. 425-429, 2010.

[60] T. Tateishi, R. Yamasaki, M. Tanaka et al., "CSF chemokine alterations related to the clinical course of amyotrophic lateral sclerosis," Journal of Neuroimmunology, vol. 222, no. 1-2, pp. 76-81, 2010.

[61] J. K. Lee, J. H. Shin, J. E. Lee, and E. J. Choi, "Role of autophagy in the pathogenesis of amyotrophic lateral sclerosis," Biochimica et Biophysica Acta (BBA) - Molecular Basis of Disease, vol. 1852, no. 11, pp. 2517-2524, 2015.

[62] J. A. Oakes, M. C. Davies, and M. O. Collins, "TBK1: a new player in ALS linking autophagy and neuroinflammation," Molecular Brain, vol. 10, no. 1, p. 5, 2017.

[63] Y. K. Moliaka, A. Grigorenko, D. Madera, and E. I. Rogaev, "Impas 1 possesses endoproteolytic activity against multipass membrane protein substrate cleaving the presenilin 1 holoprotein," FEBS Letters, vol. 557, no. 1-3, pp. 185-192, 2004. 


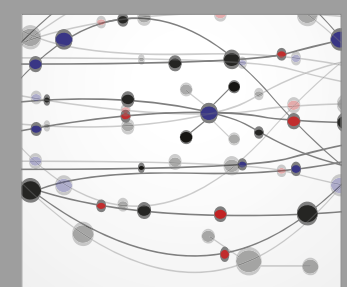

The Scientific World Journal
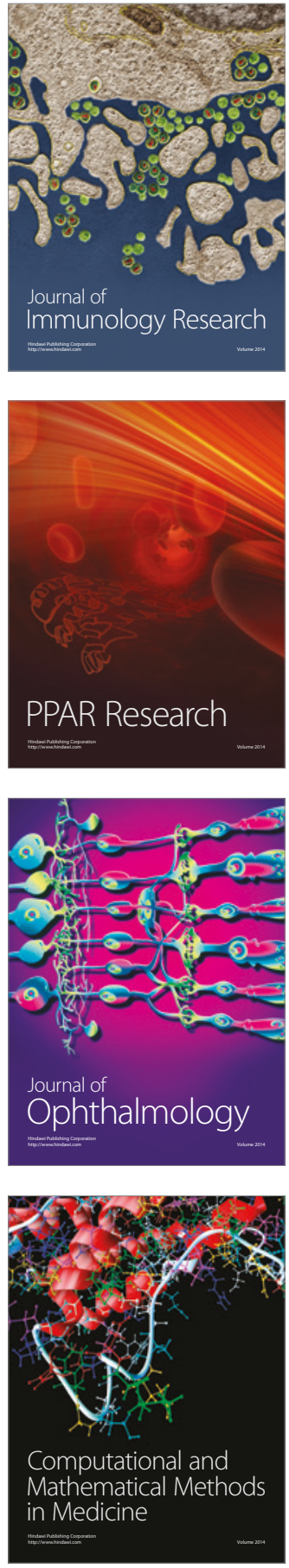

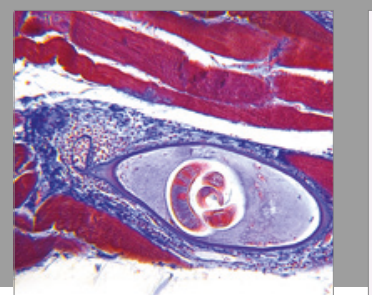

Gastroenterology Research and Practice
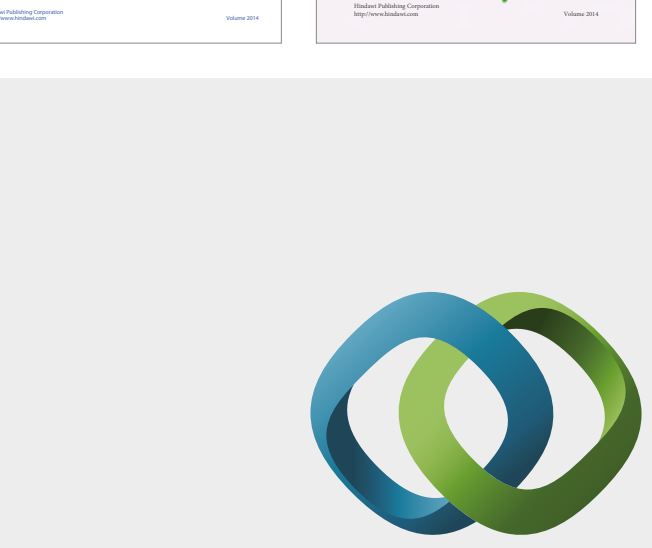

\section{Hindawi}

Submit your manuscripts at

https://www.hindawi.com
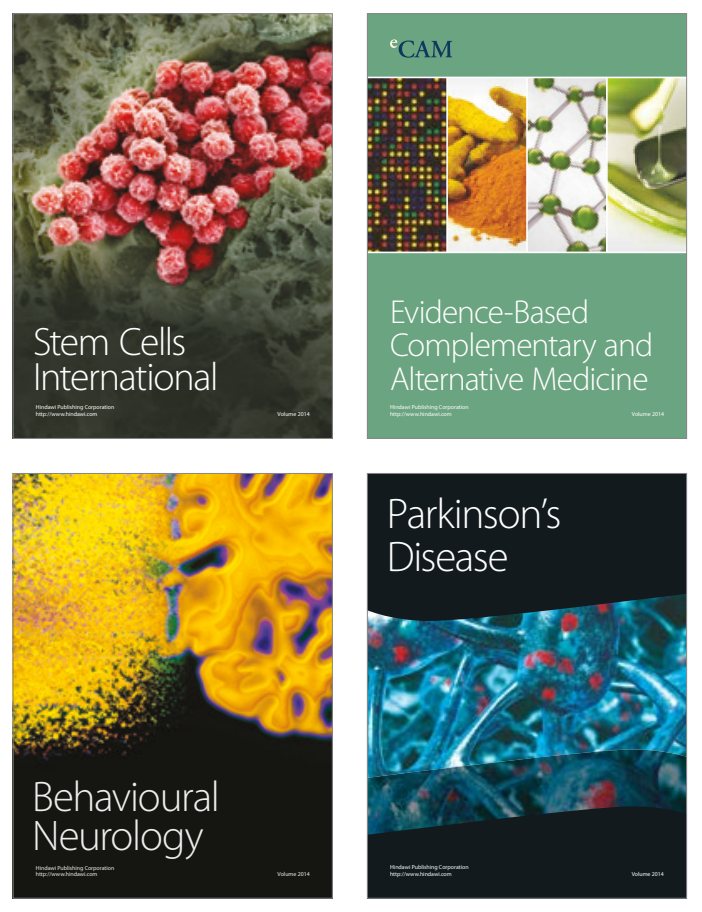
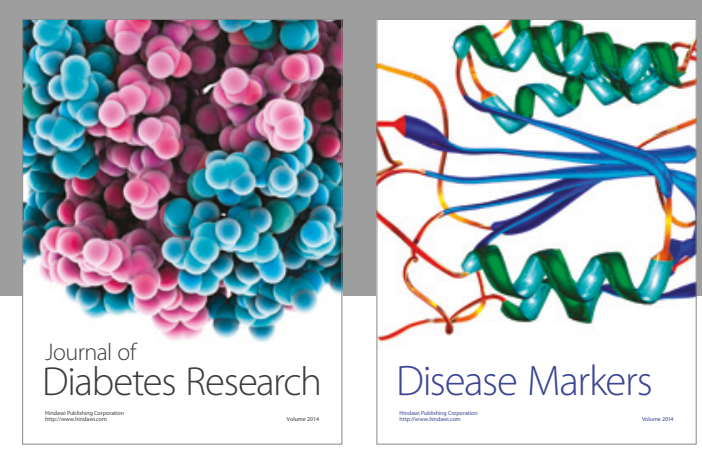

Disease Markers
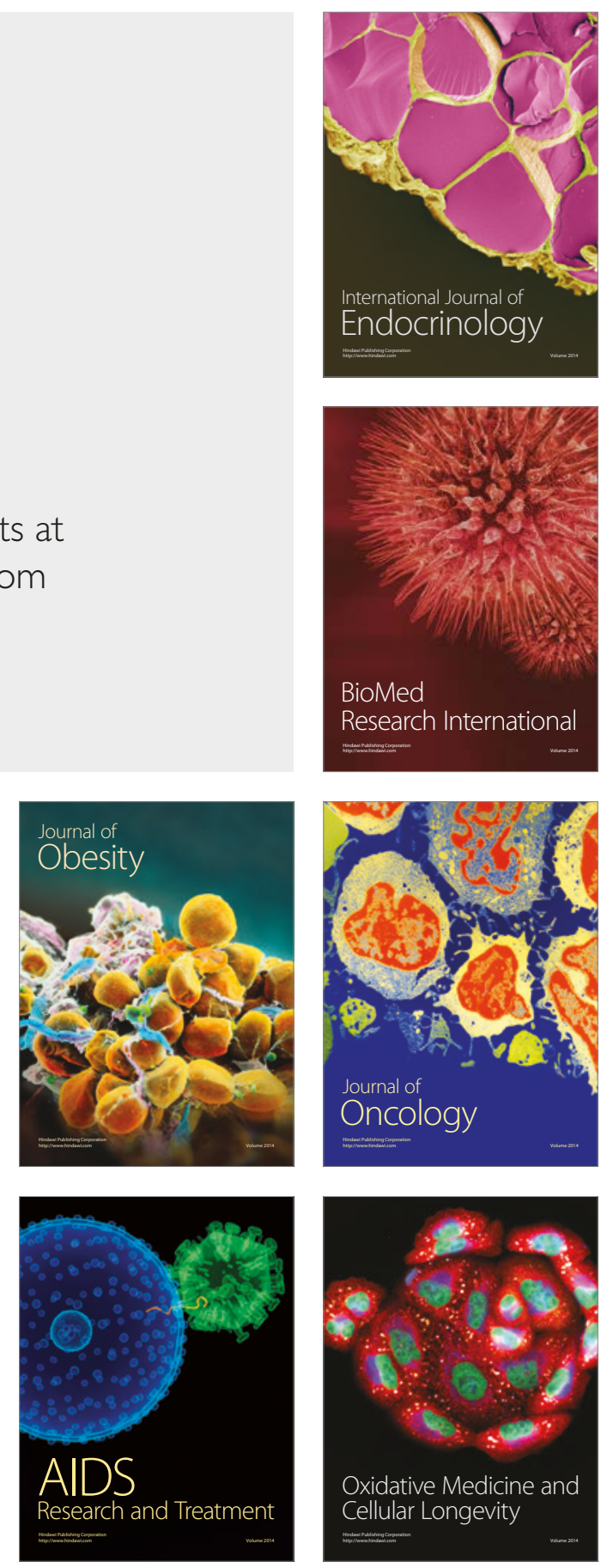